\title{
ON CONNECTED IRRESOLVABLE HAUSDORFF SPACES ${ }^{1}$
}

\author{
DOUGLAS R. ANDERSON
}

Hewitt [2, p. 327] has raised, and Padmavally [4] has answered to the affirmative, the question of the existence of connected irresolvable Hausdorff spaces. The purpose of this note is to prove a general existence theorem for connected irresolvable Hausdorff spaces which shows that the class of such spaces is more numerous than previously supposed.

In the discussion preceding Theorem 2 the underlying point set of a topological space will be denoted by $X$. A topology on $X$ will be denoted by either $R$ or $T$. When it is necessary to distinguish between different topologies on the same set $X$, subscripts will be used.

We recall some definitions and notations. If $R_{1}$ and $R_{2}$ are two topologies for $X, R_{2}$ is an expansion of $R_{1}$ if $R_{1} \subseteq R_{2}$. A topology $R$ for $X$ is irresolvable if in the space $(X, R)$ there is no dense set $D$ for which $X-D$ is also dense. The dispersion character of a topology $R$ for $X$, denoted by $\Delta(R)$, is the least cardinality of a nonempty open set in $R$.

Throughout this paper we shall be concerned with a special class of expansions of a given topology on $X$. We make the following

Definition. Let $(X, R)$ be a topological space, $\left\{D_{\alpha}: \alpha \in A\right\}$ be the set of all $R$-dense subsets of $X$. An expansion $R^{*}$ of $R$ is admissible if $R^{*}$ has a subbasis of the form $R \cup\left\{D_{\beta}: \beta \in B \subseteq A\right\}$ and if each of the sets $D_{\beta}$ is $R^{*}$-dense.

We note that any admissible expansion $R^{*}$ of $R$ has a unique subbasis $S^{*}=R \cup\left\{D_{\beta}: \beta \in B \subseteq A\right\}$ with $B$ maximal. $S^{*}=R \cup\{D: D$ is $R^{*}$-dense and $R^{*}$-open $\}$ is such a subbasis for $R^{*}$. This subbasis will be called the admissible subbasis for $R^{*}$ and the subset $B \subseteq A$ will be called the index class corresponding to $R^{*}$.

Lemma 1. Let $R^{*}$ be an admissible expansion of the connected topology $R$. Then $R^{*}$ is connected.

Proof. Suppose $R^{*}$ is not connected. Then there exist nonempty disjoint open sets $O_{1}$ and $O_{2}$ in $R^{*}$ such that $O_{1} \cup O_{2}=X$. Since $R^{*}$ is admissible, we can write

Received by the editors September 6, 1963 and, in revised form, January 11, 1964.

${ }_{1}$ This work was partially supported by the National Science Foundation through grant NSF GP-1853. 


$$
O_{1}=\bigcup_{\phi \in P}\left(O_{\phi} \cap\left(\bigcap_{i=1}^{n} D_{\phi_{i}}\right)\right), \quad O_{2}=\bigcup_{\psi \in Q}\left(O_{\psi} \cap\left(\bigcap_{j=1}^{m} D_{\psi_{j}}\right)\right),
$$

where $O_{\phi}, O_{\psi} \in R$ and $P, Q$ are index sets. But then

$$
\varnothing=O_{1} \cap O_{2}=\underset{\phi, \psi}{\cup}\left(O_{\phi} \cap O_{\psi} \cap\left(\bigcap_{i=1}^{n} D_{\phi_{i}}\right) \cap\left(\bigcap_{j=1}^{m} D_{\psi_{j}}\right)\right) .
$$

Hence, for all $\phi, \psi$ we have

$$
O_{\phi} \cap O_{\psi} \cap\left(\bigcap_{i=1}^{n} D_{\phi_{i}}\right) \cap\left(\bigcap_{j=1}^{m} D_{\psi_{j}}\right)=\varnothing .
$$

Now since $R^{*}$ is admissible

$$
O_{\phi} \cap O_{\psi} \cap\left(\bigcap_{i=1}^{n} D_{\phi_{i}}\right) \cap\left(\bigcap_{j=1}^{m-1} D_{\psi_{j}}\right)=O^{*}
$$

is $R^{*}$-open and $D_{\psi_{m}}$ is $R^{*}$-dense. Since $O^{*} \cap D_{\psi_{m}}=\varnothing$, we have $O^{*}=\varnothing$. Therefore

$$
O_{\phi} \cap O_{\psi} \cap\left(\bigcap_{i=1}^{n} D_{\phi_{i}}\right) \cap\left(\bigcap_{j=1}^{m-1} D_{\psi_{j}}\right)=\varnothing .
$$

Proceeding by induction, it follows that $O_{\phi} \cap O_{\psi}=\varnothing$ for all $\phi$ and $\psi$. But then $U_{\phi \in P} O_{\phi}$ and $U_{\psi \in Q} O_{\psi}$ are nonempty disjoint $R$-open sets which cover $X$. Hence $R$ is not connected, which is a contradiction.

REMARK. Let $X$ be a set of infinite cardinality $\tau_{0}$. Let $\tau$ be an infinite cardinal number such that $\tau_{0} \geqq \tau$. Let $F(\tau)$ be the set of all subsets of $X$ whose complement has cardinality $<\tau$. Then $\mathcal{F}(\tau)$ is a filter.

Proof. This is obvious.

Definition. Let $X$ and $\tau$ be as in the above remark and let $R$ be a topology on $X$. Then $R_{\tau}$ shall denote the topology on $X$ with subbasis $S_{\tau}=R \cup \mathcal{F}(\tau)$. Since $\mathcal{F}(\tau)$ is a filter, a basis for $R_{\tau}$ may be taken to be the family of all sets of the form $O \cap F$ where $O \in R$ and $F \in \mathcal{F}(\tau)$.

Lemma 2. Let $R$ be a connected topology with $\Delta(R) \geqq \tau$. Then $R_{\tau}$ is connected and $\Delta\left(R_{\tau}\right) \geqq \tau$.

Proof. Since $\Delta(R) \geqq \tau$, every set $F \in F(\tau)$ is $R$-dense, for no $R$-open set can be contained in $X-F$. Since it is clear that every $F \in \mathcal{F}(\tau)$ is $R_{\tau}$-dense, it follows directly from the definition of $R_{\tau}$ that $R_{\tau}$ is an admissible expansion of $R$. Hence $R_{\tau}$ is connected by Lemma 1 .

If $\Delta\left(R_{\tau}\right)<\tau$, then there is a nonempty $R_{\tau}$-open set $O$ whose cardinality is less than $\tau$. Hence $X-O \in \mathcal{F}(\tau)$ and, consequently, $X-O$ is also $R_{\tau}$-open. Thus $R_{\tau}$ is not connected, which is a contradiction.

We now proceed to the main theorem. 
THEOREM 1. Let $\tau$ be an infinite cardinal number. Let $R$ be a connected topology for $X$ with $\Delta(R) \geqq \tau$. Then there exists a connected irresolvable expansion $R^{*}$ of $R$ with $\Delta\left(R^{*}\right) \geqq \tau$.

Proof. By Lemma 2, $R_{\tau}$ is a connected expansion of $R$ with $\Delta\left(R_{\tau}\right)$ $\geqq \tau$. We shall expand $R_{\tau}$ to obtain $R^{*}$.

Let $\mathfrak{A}$ be the set of all admissible expansions $T$ of $R_{\tau}$ with the following ordering: $T_{1} \leqq T_{2}$ if $S_{1} \subseteq S_{2}$, where $S_{i}$ is the admissible subbasis for $T_{i}, i=1,2$. We note that $T_{1}<T_{2}$ if and only if there is some set $D$ which is $T_{2}$-dense and $T_{2}$-open and which is also $T_{1}$-dense, but is not $T_{1}$-open.

$\mathfrak{A}$ is not empty for $R_{\tau}$ belongs to $\mathfrak{A}$. Let $\mathfrak{l}=\left\{T_{\gamma}: \gamma \in C\right\}$ be a linearly ordered subfamily of $\mathfrak{A}$ and $B_{\gamma} \subseteq A$ be the index class corresponding to $T_{\gamma}$. Consider the topology $T^{*}$ with subbasis $S^{*}=R_{\tau} \cup\left\{D_{\delta}: \delta\right.$ $\left.\in \cup_{\gamma \in C} B_{\gamma} \subseteq A\right\}$. $T^{*}$ is an admissible expansion of $R_{\tau}$, for let $D$ be any of the dense sets $D_{\delta}$ and let $O$ be any nonempty open set in the basis for $T^{*}$ generated by $S^{*}$. Then $O=O_{1} \cap\left(\bigcap_{i=1}^{n} D_{\beta_{i}}\right)$, where $O_{1} \in R_{\tau}$. Since there are only a finite number of the sets $D, D_{\beta_{i}}, i=1, \cdots, n$, and since $\mathfrak{R}$ is linearly ordered, there exists an index $\gamma_{0}$ such that $D, D_{\beta_{i}} \in S_{\gamma_{0}}$ for $i=1, \cdots, n$. But $T_{\gamma_{0}}$ is admissible; hence $O$ is $T_{\gamma_{0}}$-open and $O \cap D \neq \varnothing$. But $O$ was an arbitrary element of a basis for $T^{*}$. Hence $D$ is $T^{*}$-dense and $T^{*}$ is admissible. Thus every linearly ordered subfamily of $\mathfrak{A}$ has an upper bound in $\mathfrak{A}$; hence, by Zorn's Lemma, $\mathfrak{A}$ has a maximal element $R^{*}$ with admissible subbasis $S^{*}$ $=R_{\tau} \cup\left\{D_{\beta}: \beta \in B^{*}\right\}$.

$R^{*}$ is irresolvable, for if it is not, there is an $R^{*}$-dense set $D$ such that $X-D$ is also $R^{*}$-dense. Hence $D$ is not $R^{*}$-open. But $D$ is dense in $R_{\tau}$ and the topology $T$ with subbasis $S^{*} \cup\{D\}$ is an admissible expansion of $R_{\tau}$ strictly greater than $R^{*}$. This contradicts the maximality of $R^{*}$.

Since $R^{*}$ is an admissible expansion of $R_{\tau}$ and $R_{\tau}$ is connected, $R^{*}$ is connected. Since $R^{*}$ is a connected expansion of $R_{\tau}, \Delta\left(R^{*}\right) \geqq \tau$, by the same argument used in Lemma 2 to show $\Delta\left(R_{\tau}\right) \geqq \tau$. Clearly $R^{*}$ is an expansion of $R$.

Hence $R^{*}$ is the desired topology.

CoROllary. Let $R$ be a connected Hausdorff topology for $X$ with $\Delta(R) \geqq \tau$, where $\tau$ is an infinite cardinal number. Then there exists a connected irresolvable Hausdorff expansion $T$ of $R$ with $\Delta(T) \geqq \tau$.

Proof. Since the property of being Hausdorff is invariant under expansions, we may apply Theorem 1 to $R$ and let $T$ be the topology $R^{*}$.

As a consequence of Theorem 1, to establish the existence of connected irresolvable Hausdorff spaces with arbitrary infinite disper- 
sion character, it suffices to establish the existence of connected Hausdorff spaces of arbitrary infinite dispersion character. The existence of the latter spaces is established by

TheOREM 2. Let $\tau$ be an infinite cardinal number. Then there exists a connected Hausdorff space $(X, R)$ with $\Delta(R)=\tau$.

Proof. For $\tau=\aleph_{0}$, let $(X, R)$ be any countable connected Hausdorff space. (Examples of such spaces are well known. See [5] or [1].) Then $\Delta(R)=\boldsymbol{\aleph}_{0}$, for, if not, there is a nonempty finite open set $O$. But since $R$ is Hausdorff, the finite set $O$ is closed. Thus since $O$ is both open and closed in $R, R$ is not connected, which is a contradiction.

For $\tau>\aleph_{0}$, let $C$ be a countable connected Hausdorff space, let $T$ be a set of cardinality $\tau$, and let $p: T \rightarrow C$ be any function. Then the set $X$ of all functions $q: T \rightarrow C$ which agree with $p$ at all but a finite number of points with the topology $R$ of pointwise convergence (see $[3$, p. 217]) is the desired space $(X, R)$. For clearly $R$ is connected, Hausdorff, and $\Delta(R) \geqq \tau$. But also each function in $X$ is determined by its behavior on a finite subset $F$ of $T$ and for each such subset $F$, there are only $\boldsymbol{\aleph}_{0}$ ard $\boldsymbol{F}=\boldsymbol{\aleph}_{0}$ possible functions. Since card $T=\tau$, there are $\tau$ finite subsets of $T$. Hence there are $\aleph_{0} \cdot \tau=\tau$ points in $X$. Thus $\Delta(R) \leqq \tau$ and the last assertion of the theorem is established.

Combining the corollary above with Theorem 2 we obtain

COROLLARY. Let $\tau$ be an infinite cardinal number. Then there exists a connected irresolvable Hausdorff space $(X, R)$ with $\Delta(R)=\tau$.

If the above results are applied to the real line, we see that there is a connected topology finer than the usual topology with the property that each nonempty open set has c points in which $U$ and the complement of $U$ are never simultaneously dense.

The author would like to express his thanks to Professor Hing Tong for his assistance in preparing the manuscript, and to the referee for his helpful suggestions.

\section{REFERENCES}

1. R. H. Bing, A connected countable Hausdorff space, Proc. Amer. Math. Soc. 4 (1953), 474.

2. E. Hewitt, $A$ problem of set theoretic topology, Duke Math. J. 10 (1943), 309-333.

3. J. L. Kelley, General topology, Van Nostrand, Princeton, N. J., 1955.

4. K. Padmavally, An example of a connected irresolvable Hausdorff space, Duke Math. J. 20 (1953), 513-520.

5. P. Urysohn, Über die Mächtigkeit der zusammenhängenden Menge, Math. Ann. 94 (1925), 262-295.

\section{YALE UNIVERSITY}

